\title{
Frecuencia y características tomográficas del daño al nervio óptico en sujetos con glaucoma
}

\section{Frequency and tomographic features of optic nerve injury in glaucoma}

\author{
Óscar Olmedo-Alonso ${ }^{1}$, Dulce M. Razo-Blanco Hernández ${ }^{2}$, Selma A. Somilleda-Ventura ${ }^{3}$ y \\ VIRGILIO LIMA-GÓMEZ ${ }^{1 *}$ \\ ${ }^{1}$ Servicio de Oftalmología, Hospital Juárez de México; ${ }^{2}$ División de Investigación, Hospital Juárez de México; ${ }^{3}$ Centro de Investigación Biomédica, Hospital Nuestra \\ Señora de la Luz. Ciudad de México, México
}

\begin{abstract}
Glaucoma is an optic neuropathy which causes visual field loss; although the distribution of visual field loss is well characterized, the frequency of tomographic optic nerve damage in glaucoma is unknown in our media. Methods: observational, descriptive, cross-sectional, and retrospective study. We included subjects aged 40-70 years and measured the peripapillary nerve fiber layer thickness with optical coherence tomography. Patients were grouped according to the presence of decreased thickness: group 1 (with fiber loss), group 2 (with borderline loss), and group 3 (without loss). We determined the frequencies of decreased thickness per group and compared them between groups (Student's t test). Results: 115 subjects: 22 in group 1, 25 in group 2, and 68 in group 3. The superior and inferior sectors were more often affected; thickness decreased more frequently in meridians 1, 2, 5, 6,7 , and $10(p<0.001)$. Conclusions: $19.1 \%$ of the sample had nerve fiber layer thinning, more commonly at the superior or inferior sectors, where tomographic evaluation could focus to detect early damage.
\end{abstract}

Key words: Nerve fiber layer. Optical coherence tomography. Glaucoma.

\section{RESUMEN}

Antecedentes: El glaucoma es una neuropatía óptica que causa pérdida del campo visual, que está bien caracterizada; en nuestro medio se desconoce la frecuencia de daño tomográfico al nervio óptico por glaucoma. Métodos: Estudio observacional, descriptivo, transversal y retrospectivo. Incluimos sujetos de 40-70 años, y medimos el grosor de la capa de fibras nerviosas peripapilar con tomografía de coherencia óptica.Se formaron grupos de acuerdo con la presencia de grosor disminuido: grupo 1 (con pérdida de fibras), grupo 2 (en el límite) y grupo 3 (sin pérdida). Se determinaron las frecuencias de grosor disminuido por grupo y se compararon entre grupos (t de Student). Resultados: 115 sujetos, 22 del grupo 1, 25 del 2 y 68 del 3. Se afectaron más comúnmente los sectores superior e inferior; el grosor disminuyó más frecuentemente en los meridianos 1, 2, 5, 6, 7 y 10 ( $p<0.001)$ Conclusiones: $19.1 \%$ de la muestra tuvo adelgazamiento de la capa de fibras nerviosas, más comúnmente superior e inferior, donde podría enfocarse la evaluación tomográfica para detectar daño temprano.

Palabras clave: Tomografía de coherencia óptica. Capa de fibras nerviosas. Glaucoma.

\section{Correspondence:}

*Virgilio Lima-Gómez

E-mail: forscher7@gmail.com
Date of reception: 09-12-2019

Date of acceptance: 21-09-2020

DOI: 10.24875/HMCM.19000253
Available online: 18-11-2020 Hosp Med Clin Manag. 2020;13:109-15

2604-0018 / @ 2020 Mexican Regional Hospitals of High Specialty and Federal Hospitals. Published by Permanyer. This is an open access article under the CC BY-NC-ND license (http://creativecommons.org/licenses/by-nc-nd/4.0/). 


\section{INTRODUCCIÓN}

El glaucoma es una neuropatía óptica progresiva caracterizada por cambios estructurales en la cabeza del nervio óptico, pérdida de las células ganglionares retinianas y de sus axones ${ }^{1}$, con el adelgazamiento de la capa de fibras nerviosas de la retina (RNFL, retinal nerve fiber layer), cuyo resultado final condiciona una pérdida del campo visual ${ }^{2}$.

Es una de las principales causas de ceguera irreversible en el mundo. Se estima una prevalencia global del 3.54\% (intervalo de confianza [IC] 95\%: 2.09-5.82), del $3.05 \%$ para glaucoma de ángulo abierto (IC 95\%: 1.695.27 ) y del $0.50 \%$ para glaucoma de ángulo cerrado (IC 95\%: $0.11-1.36)^{3}$.

En la exploración de fondo de ojo los cambios estructurales que hacen sospechar la enfermedad pueden ser agrandamiento difuso o local de la copa óptica, desplazamiento, restricción y variación en el calibre de los vasos papilares, hemorragias en el disco óptico, defectos en la RNFL, atrofia peripapilar y elevación de la presión intraocular ${ }^{4}$.

En la actualidad, los nuevos equipos tecnológicos permiten evaluar de manera objetiva el nervio óptico y la RNFL, como es el caso de la tomografía de coherencia óptica $(\mathrm{TCO})^{5}$ y la polarimetría láser de barrido, que proporcionan estimaciones cuantitativas del grosor de la RNFL, así como la oftalmoscopia láser de barrido confocal, que evalúa la topografía del nervio óptico, aunque también puede proporcionar estimaciones indirectas de la integridad de la RNFL 4 .

Ante un caso sospechoso, toda evaluación clínica se complementa con una TCO del nervio óptico y de la RNFL, para las cuales se requiere de medios ópticos transparentes (córnea, cristalino y vítreo) para la toma, y un examen de campos visuales ${ }^{4}$, que requiere la cooperación del paciente y buena fijación ${ }^{6}$. En ojos sanos, la detección mediante la TCO tiene una sensibilidad excelente (> 99\%), al igual que para detectar los cambios moderados en el nervio óptico de los pacientes con sospecha de glaucoma (96.4\%) $)^{1}$. En los pacientes con daño inicial el grosor de RNFL es el mejor parámetro para predecir el daño, especialmente en el sector inferior temporal, con una sensibilidad del $71.4 \%$ y una especificidad del $95 \%$, superando así los parámetros en la evaluación clínica del nervio óptico ${ }^{5,6}$.
La pérdida de las células ganglionares es el evento primario de la enfermedad ${ }^{7}$ y algunos estudios muestran que este cambio precede la pérdida funcional hasta en cinco años ${ }^{8,9}$, reportando que se pierde el 30-35\% de las células ganglionares de la retina antes de que aparezca una anomalía en la perimetría estándar²,10.

Los parámetros de la RNFL son significativamente inferiores para diagnosticar incluso glaucoma perimétrico. Adicionalmente, la prueba de simetría de la RNFL, un algoritmo de detección que compara los segmentos correspondientes de la mitad superior con la inferior, tiene una sensibilidad del 95\% y una especificidad del $100 \%$ para distinguir sujetos con glaucoma ${ }^{11}$. Sin embargo, en etapas más avanzadas el progreso del daño glaucomatoso se detecta mejor por cambios en el campo visual, ya que la variación del grosor de la RNFL es muy pequeña para ser detectada y la variación del campo visual es mayor y fácilmente detectada ${ }^{10}$. Tradicionalmente se ha descrito que en el glaucoma los sectores más afectados son el inferior, inferior temporal, superior y superior temporal. Sin embargo, se desconoce la frecuencia y características tomográficas del daño al nervio óptico en sujetos con glaucoma en nuestro medio; conocerlas permitiría definir el sector al que debe dirigirse la atención al momento del estudio.

\section{MÉTODOS}

Se realizó un estudio observacional, descriptivo, transversal y retrospectivo en sujetos de la Ciudad de México y su área metropolitana. La muestra se obtuvo de sujetos que acudieron por primera vez al servicio de oftalmología de un hospital federal de referencia en el periodo comprendido entre marzo y junio del 2018. El estudio fue autorizado por el comité de investigación y ética en investigación del lugar en donde se desarrolló.

Se incluyeron sujetos entre 40 y 70 años de edad sin diagnóstico oftalmológico previo, de cualquier sexo, con medios ópticos transparentes que permitieran obtener un estudio de adecuada calidad y con firma de consentimiento informado por escrito. Se excluyeron aquellos casos en los que se determinó patología del nervio óptico diferente a glaucoma.

Las variables de estudio que se tomaron para el estudio fueron todas las mediciones automáticas realizadas por la TCO: 
- Daño tomográfico por glaucoma, el cual se definió como grosor de la RNFL cuya probabilidad de ser normal se encuentre por debajo del percentil 5, de acuerdo con la base normativa de la TCO.

- Grosor de la RNFL promedio, definido por el grosor en micras de los axones de células ganglionares retinianas no mielinizadas.

- Área del borde, definido por el área en milímetros cuadrados del borde neurorretiniano.

- Área del disco, definido por la superficie medida en milímetros cuadrados dentro de los límites externos del anillo neurorretiniano.

- Proporción copa-disco (C/D) promedio, definida por el índice de la relación entre la porción papilar libre de fibras nerviosas que se sitúa por dentro del anillo neurorretiniano y el área total del disco óptico.

- Proporción C/D vertical, definida por el índice de la relación entre la porción papilar libre de fibras nerviosas que se sitúa por dentro del anillo neurorretiniano de forma vertical y el área vertical del disco óptico.

- Volumen de la copa, definido por la porción papilar libre de fibras nerviosas que se sitúa por dentro del anillo neurorretiniano.

- La simetría de la RNFL, definida por el porcentaje de correspondencia entre ambos ojos del grosor de la RNFL.

- Grosor por cuadrantes y meridianos (M) de la RNFL, que se definió por la medición del grosor en micras por cuadrante y $\mathrm{M}$ de los axones de las células ganglionares retinianas no mielinizadas.

Los sujetos con daño tomográfico por debajo del percentil 2 se asignaron al grupo 1 (sujetos con glaucoma por atrofia de fibras nerviosas); los que lo presentaban a valores en el límite, es decir, entre los percentiles 2 y 5 , se asignaron al grupo 2 (limítrofe); y los que no presentaban alteraciones en el estudio se asignaron al grupo 3 (sin glaucoma). Un investigador obtuvo los datos de los pacientes, mientras que otro investigador tomó las fotografías de fondo de ojo con cámara no midriática (Canon); un tercer investigador adquirió las imágenes de TCO utilizando el equipo Cirrus 5000 ${ }^{a}$ (Zeiss, Meditec) con las siguientes características: un cubo de nervio óptico de 200 × 200 para medir el grosor de la RNFL y de la cabeza del nervio óptico. Un cuarto investigador oftalmólogo certificado evaluó cada uno de los estudios.

Se determinó la proporción e intervalos de confianza (IC) del 95\% de los sujetos de la muestra que presentaban daño tomográfico por glaucoma, así como la proporción e IC del 95\% de sujetos con daño tomográfico en cada cuadrante y en cada $\mathrm{M}$ del nervio óptico. Los datos obtenidos fueron almacenados y analizados con el programa SPSS versión 21 para Windows.

\section{RESULTADOS}

Se evaluaron 115 sujetos, los cuales se dividieron de acuerdo con lo establecido como daño tomográfico. Al grupo 1 se asignaron 22 sujetos con datos de glaucoma, al grupo 2 se asignaron 25 sujetos que tuvieron características limítrofes, y el grupo 3 se conformó por 68 sujetos sin ninguna característica patológica en la tomografía.

En sujetos del grupo 1 la edad promedio fue de $54.36 \pm$ desviación estándar (DE) 15.16, con un intervalo de 19 a 80 años; 10 sujetos (45.5\%) fueron del sexo femenino. En el grupo 2 el promedio de edad fue de $55.52 \pm 10.82$ años; predominó el sexo femenino, con un $61.8 \%(n=42)$. En el grupo 3 el promedio de edad fue de $53.97 \pm 15.70$ ( 19 a 86 años) y 42 sujetos $(61.8 \%)$ fueron del sexo femenino.

Según las características del nervio óptico, solo difirió entre los grupos el grosor de la RNFL, lo que concuerda con los reportes previos, identificándose como el primer dato que podemos encontrar en los pacientes con glaucoma, que es la pérdida de fibras nerviosas ( $p$ $<0.001)$. El resto de las características no difirió entre los grupos (Tabla 1).

Una vez identificada la disminución de la RNFL por grupo se realizó una estratificación con base en los sectores que da la TCO, donde se identificó una diferencia significativa en los cuadrantes superior e inferior en ambos ojos $(p<0.001)$ y solo en el izquierdo en el sector temporal $(p=0.008)$ (Fig. 1). En el resto de los cuadrantes no difirieron los valores promedio en cada grupo.

También realizamos una estratificación por $\mathrm{M}$, encontrando una afección con diferencia significativa en los 
Tabla 1. Características del nervio óptico en los grupos

\begin{tabular}{|c|c|c|c|c|c|c|c|c|}
\hline \multirow[t]{2}{*}{ Variable } & \multicolumn{4}{|c|}{ 0jo derecho } & \multicolumn{4}{|c|}{ Ojo izquierdo } \\
\hline & Grupo $1 \pm D E$ & Grupo $2 \pm D E$ & Grupo $3 \pm D E$ & p & Grupo $1 \pm D E$ & Grupo $2 \pm D E$ & Grupo $3 \pm D E$ & $\mathrm{p}$ \\
\hline Grosor RNFL promedio $(\mu \mathrm{m})$ & $88.13 \pm 18.02$ & $90.92 \pm 9.46$ & $101.10 \pm 10.15$ & $<0.001$ & $86.68 \pm 17.38$ & $92.16 \pm 7.99$ & $101.04 \pm 9.85$ & $<0.001$ \\
\hline Área de borde $\mathrm{mm}^{2}$ & $1.3 \pm 60.43$ & $1.26 \pm 0.33$ & $1.34 \pm 0.2$ & 0.63 & $1.23 \pm 0.34$ & $1.27 \pm 0.27$ & $1.40 \pm 0.24$ & 0.02 \\
\hline Área de disco mm² & $2.08 \pm 0.45$ & $2.05 \pm 0.39$ & $2.06 \pm 0.46$ & 0.97 & $2.06 \pm 0.39$ & $2.02 \pm 0.36$ & $2.12 \pm 0.41$ & 0.52 \\
\hline Proporción C/D promedio & $0.58 \pm 0.18$ & $0.57 \pm 0.2$ & $0.55 \pm 0.13$ & 0.79 & $0.58 \pm 0.17$ & $0.55 \pm 0.19$ & $0.55 \pm 0.11$ & 0.65 \\
\hline Proporción C/D vertical & $0.57 \pm 0.18$ & $0.54 \pm 0.19$ & $0.53 \pm 0.12$ & 0.66 & $0.56 \pm 0.17$ & $0.51 \pm 0.18$ & $0.52 \pm 0.11$ & 0.5 \\
\hline Volumen copa mm³ & $0.22 \pm 0.18$ & $42.96 \pm 213.55$ & $0.21 \pm 0.18$ & 0.16 & $54.56 \pm 236.93$ & $0.24 \pm 0.21$ & $0.18 \pm 0.15$ & 0.09 \\
\hline
\end{tabular}

DE: desviación estándar; RNFL: fibras nerviosas de la retina (retinal nerve fiber layer); C/D: copa/disco.
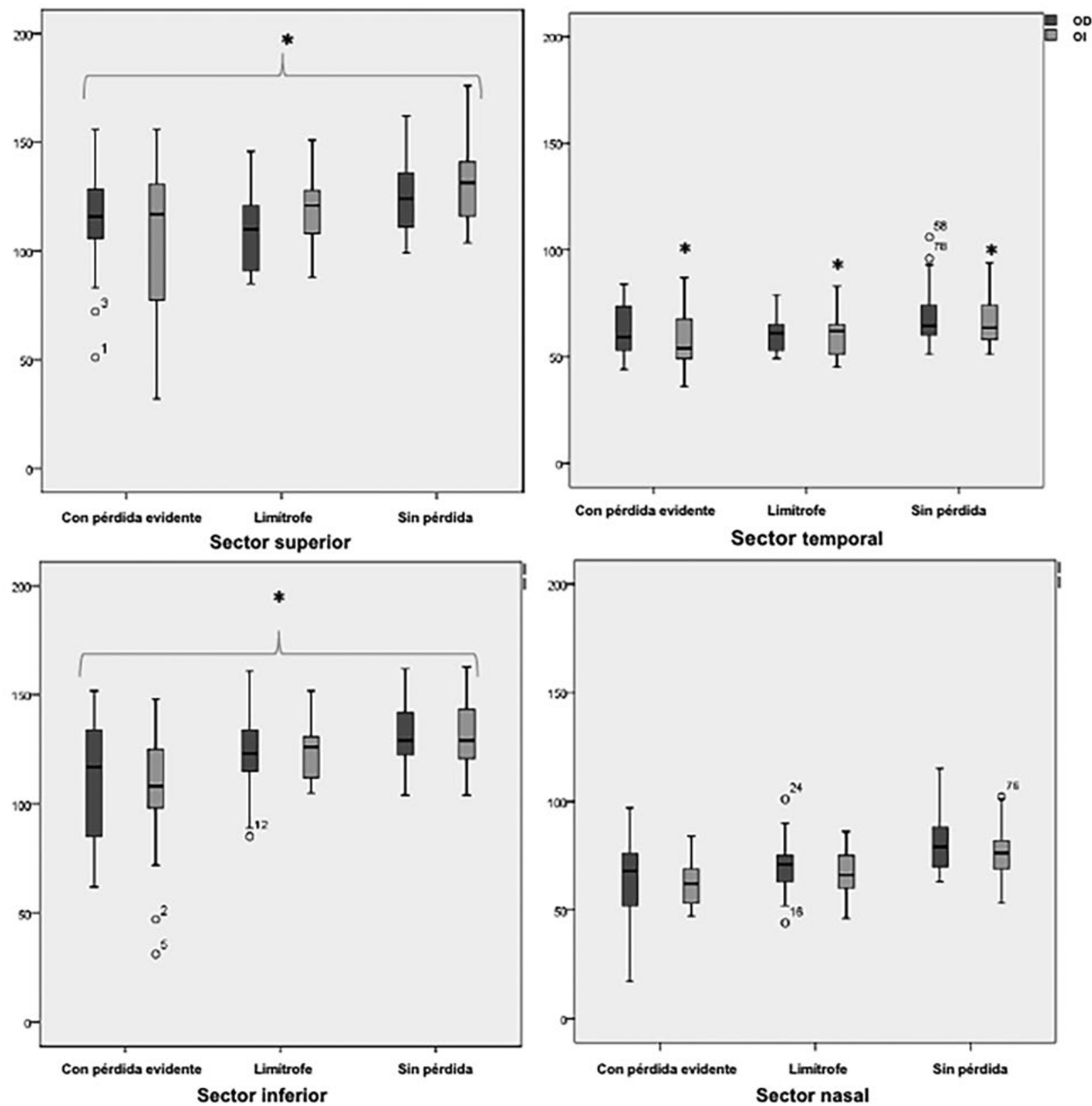

Figura 1. *p < 0.05 OD: ojo derecho Ol: ojo izquierdo 
Tabla 2. Promedio del grosor de RNFL por meridianos en los diferentes grupos

\begin{tabular}{|c|c|c|c|c|c|c|c|c|}
\hline \multirow[t]{2}{*}{ Meridiano } & \multicolumn{4}{|c|}{ 0jo derecho } & \multicolumn{4}{|c|}{ Ojo izquierdo } \\
\hline & Grupo $1 \pm D E$ & Grupo $2 \pm D E$ & Grupo $3 \pm D E$ & p & Grupo $1 \pm D E$ & Grupo $2 \pm D E$ & Grupo $3 \pm D E$ & p \\
\hline 1 & $104.13 \pm 42.24$ & $93.56 \pm 24.14$ & $109.1 \pm 19.47$ & 0.045 & $92.84 \pm 35.91$ & $125.2 \pm 29.71$ & $134.38 \pm 21.14$ & $<0.001$ \\
\hline 2 & $88.36 \pm 36.14$ & $87.48 \pm 17.65$ & $104.64 \pm 20.52$ & 0.002 & $63.68 \pm 16.27$ & $74.96 \pm 14.65$ & $79.98 \pm 14.19$ & $<0.001$ \\
\hline 3 & $59.27 \pm 33.01$ & $56.92 \pm 10.57$ & $63.85 \pm 13.27$ & 0.22 & $50.73 \pm 12.60$ & $51 \pm 9.84$ & $53.92 \pm 9.74$ & 0.32 \\
\hline 4 & $68.22 \pm 25.35$ & $66 \pm 15.37$ & $73.7 \pm 15.62$ & 0.13 & $59.42 \pm 21.72$ & $54.96 \pm 10.49$ & $64.17 \pm 14.6$ & 0.03 \\
\hline 5 & $96.27 \pm 30.43$ & $105.56 \pm 16.95$ & $114.97 \pm 26.86$ & 0.01 & $103.57 \pm 33.07$ & $120.68 \pm 20.41$ & $130.9 \pm 19.54$ & $<0.001$ \\
\hline 6 & $116.13 \pm 39.43$ & $144.8 \pm 29.39$ & $148.82 \pm 26.86$ & $<0.001$ & $116.78 \pm 36.87$ & $146.08 \pm 19.01$ & $153.57 \pm 24.45$ & $<0.001$ \\
\hline 7 & $108.9 \pm 33.47$ & $117.28 \pm 22.07$ & $134.11 \pm 22.38$ & $<0.001$ & $91.73 \pm 42.98$ & $104.36 \pm 17.01$ & $114.34 \pm 20.28$ & 0.003 \\
\hline 8 & $68.81 \pm 26.08$ & $56.64 \pm 10.37$ & $66.56 \pm 14.27$ & 0.02 & $65.94 \pm 45.98$ & $65.76 \pm 17.07$ & $72.25 \pm 20.28$ & 0.38 \\
\hline 9 & $57.04 \pm 20.27$ & $50.28 \pm 8.2$ & $54.76 \pm 10.56$ & 0.16 & $63 \pm 47.5$ & $54.28 \pm 8.44$ & $58.36 \pm 10.16$ & 0.41 \\
\hline 10 & $69.54 \pm 18.28$ & $75.44 \pm 13.31$ & $80.32 \pm 14.58$ & 0.01 & $79.52 \pm 23.15$ & $80.76 \pm 14.9$ & $99.28 \pm 21.9$ & $<0.001$ \\
\hline 11 & $105.68 \pm 27.98$ & $116.12 \pm 18.23$ & $133.22 \pm 23.84$ & $<0.001$ & $112.84 \pm 45.88$ & $110 \pm 24.65$ & $120.8 \pm 21.09$ & 0.20 \\
\hline 12 & $115.68 \pm 44.44$ & $119.12 \pm 28.38$ & $126.91 \pm 25.90$ & 0.26 & $111.15 \pm 50.21$ & $116 \pm 23.85$ & $134.25 \pm 21.8$ & 0.003 \\
\hline
\end{tabular}

DE: desviación estándar; RNFL: fibras nerviosas de la retina (retinal nerve fiber layer).

M 1, 2, 5, 6, 7 y 10. El M1 corresponde a parte del sector superior; el M2 corresponde en ojos derechos al sector nasal, pero en los ojos izquierdos al temporal; en el ojo izquierdo, el M4, que también tuvo una diferencia significativa, corresponde al mismo sector que el M2. Todos los $\mathrm{M}$ del sector inferior presentaron diferencia significativa en ambos ojos. En el M8 solo del ojo derecho, que corresponde al sector temporal, se encontraba con diferencia significativa entre los grupos. El M10, afectado en ambos ojos, de un lado correspondía al sector nasal y del otro al sector temporal (Tabla 2).

Al realizar el análisis en cuanto a la frecuencia de los cuadrantes afectados en los grupos 1 y 2 se encontró que, aunque en algún momento se afectaba algún sector, el superior e inferior fueron los que más frecuentemente estaban involucrados (Fig. 2).

En el caso de los $M$, en el grupo 1 fue más frecuente encontrar pérdida de fibras nerviosas en M1 y M11, que ambos corresponden al sector superior. En el caso del grupo 2 los M más frecuentemente afectados fueron el 1 y el 8, que corresponden al sector superior y al sector nasal, respectivamente (Tabla 3).

\section{DISCUSIÓN}

El daño tomográfico al nervio óptico se encontró en el 19.13\% de la muestra (IC 95\%: 11.9-26.3), lo cual supera la prevalencia de glaucoma en el mundo. El sector superior, así como el M1, son las partes más afectadas
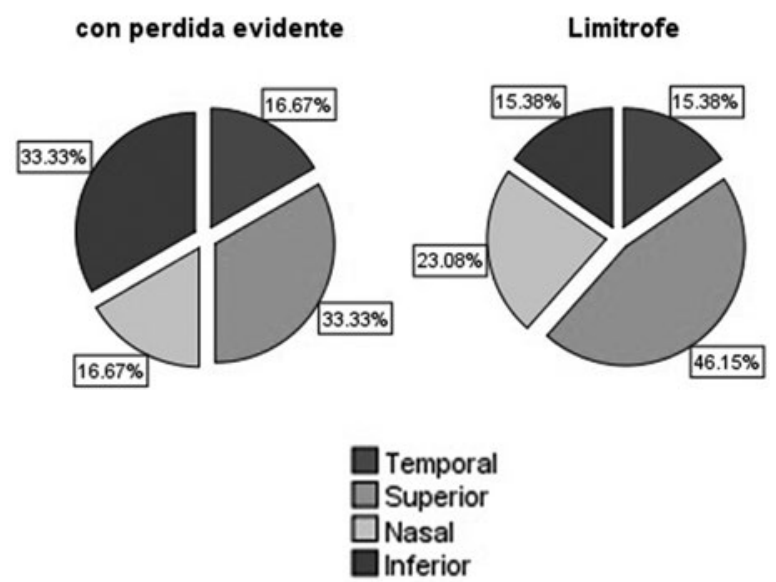

Figura 2. Distribución del daño tomográfico por cuadrantes.

significativamente del nervio óptico, aunque se puede afectar cualquier zona.

El grosor de la RNFL se compara según edad, raza y tamaño del disco y los resultados se pueden representar ya sea con un mapa de colores o una onda sinusoidal que representa el grosor peripapilar $360^{\circ}{ }^{12}$. Además, se reporta cuantitativamente en cada sector, por lo que se ha definido en pacientes con glaucoma un grosor de RNFL promedio de $69.2 \pm 17.1 \mu$, y en cada sector: temporal $52.6 \pm 15.1 \mu$, superior $84.5 \pm 23.5 \mu$, nasal $57.4 \pm 13.8 \mu$ e inferior $81.0 \pm 28.2 \mu^{7}$. Esto difiere significativamente de lo encontrado en este estudio, en que fueron mayores $(p<0.05)$ en todos los sectores, pero sin llegar a los encontrados en población sin glaucoma. 
Tabla 3. Frecuencia de daño tomográfico al nervio óptico, por TCO

\begin{tabular}{|c|c|c|c|c|c|c|c|}
\hline & Meridiano & n (\%) & IC $95 \%$ & & Meridiano & n (\%) & IC $95 \%$ \\
\hline \multirow[t]{12}{*}{ Grupo 1} & 1 & 14 (63.6) & $43.5-83.7$ & Grupo 2 & 1 & $10(40)$ & $20.8-59.2$ \\
\hline & 2 & $8(36.4)$ & $16.3-56.5$ & & 2 & $3(12)$ & $0-24.7$ \\
\hline & 3 & $6(27.3)$ & $8.7-45.9$ & & 3 & $3(12)$ & $0-24.7$ \\
\hline & 4 & $6(27.3)$ & $8.7-45.9$ & & 4 & $4(16)$ & $1.6-30.4$ \\
\hline & 5 & $7(31.8)$ & $12.3-51.3$ & & 5 & $2(8)$ & 0-18.6 \\
\hline & 6 & $11(50)$ & $29.1-70.9$ & & 6 & $1(4)$ & $0-11.7$ \\
\hline & 7 & $8(36.4)$ & $16.3-56.5$ & & 7 & $1(4)$ & 0-11.7 \\
\hline & 8 & $5(22.7)$ & $5.2-40.2$ & & 8 & 7 (28) & $10.4-45.6$ \\
\hline & 9 & $3(13.6)$ & $0-27.9$ & & 9 & $3(12)$ & $0-24.7$ \\
\hline & 10 & $8(36.4)$ & $16.3-56.5$ & & 10 & $3(12)$ & $0-24.7$ \\
\hline & 11 & $8(36.4)$ & $16.3-56.5$ & & 11 & $4(16)$ & $1.6-30.4$ \\
\hline & 12 & 7 (31.8) & $12.3-51.3$ & & 12 & $2(8)$ & 0-18.6 \\
\hline
\end{tabular}

TCO: tomografía de coherencia óptica; IC: intervalo de confianza.

La evaluación clínica de la papila es relativamente subjetiva, en donde se identifican determinadas características del nervio óptico que preceden a los cambios en el campo visual, así que la medición de la RNFL permite identificar pequeños cambios de manera objetiva, lo que da la opción de iniciar tempranamente con el tratamiento y con ello retrasar la pérdida visual en estos pacientes.

- Se ha reportado en otros estudios que los sectores más afectados son los inferiores y superiores ${ }^{6}$, lo cual concuerda con el presente estudio; sin embargo, no se pueden excluir a los otros cuadrantes debido a que también pueden estar afectados. La importancia del grosor de la RNFL no se puede subestimar, porque en teoría el adelgazamiento de esta es el cambio estructural que aparece más tempranamente y está directamente relacionado con la pérdida de células ganglionares retinianas ${ }^{10}$. Sin embargo, cabe recordar que para poder realizar este estudio es necesaria la cooperación del sujeto y que exista una buena fijación al realizarlo, además de complementarlo con la evaluación clínica y de los campos visuales para tener un mayor control de los pacientes.

Algunos estudios han evaluado el grosor de la RNFL y su relación con el diagnóstico de glaucoma o con la pérdida de campo visual. Los cuadrantes superior e inferior se han caracterizado por tener una mayor confiabilidad para identificar los ojos con pérdida del campo visual por glaucoma (área bajo la curva; 0.79-0.952, superior; 0.863-0.971, inferior) en comparación con los controles $^{13}$, cuyos resultados son similares a los nuestros, en donde difieren significativamente los valores en los sectores superior e inferior en comparación con los controles reportados previamente.

Una de las limitaciones de este estudio es que solo se contó con el estudio de TCO junto con la evaluación clínica del nervio óptico y no se realizó una valoración de campos visuales; sin embargo, el objetivo del estudio solo fue la evaluación de las características encontradas únicamente mediante el estudio de TCO. Una de las fortalezas del estudio es que, a pesar de ser una muestra reducida, se encontró una frecuencia alta de pacientes con datos de glaucoma, por lo cual se pudieron estudiar estas características.

\section{CONCLUSIONES}

Aunque fue más frecuente encontrar daño tanto en los $\mathrm{M}$ que corresponden al sector superior como en este mismo sector, puede afectarse cualquier parte del nervio óptico, por lo que deben revisarse completamente de forma estandarizada los nervios ópticos y la RNFL para evitar subestimar los daños.

\section{FINANCIAMIENTO}

La presente investigación no ha recibido ninguna beca específica de agencias de los sectores público, comercial o sin ánimo de lucro.

\section{CONFLICTO DE INTERESES}

Los autores declaran no tener conflicto de intereses. 


\section{RESPONSABILIDADES ÉTICAS}

Protección de personas y animales. Los autores declaran que para esta investigación no se han realizado experimentos en seres humanos ni en animales.

Confidencialidad de los datos. Los autores declaran que han seguido los protocolos de su centro de trabajo sobre la publicación de datos de pacientes.

\section{Derecho a la privacidad y consentimiento informa-}

do. Los autores han obtenido el consentimiento informado de los pacientes y/o sujetos referidos en el artículo. Este documento obra en poder del autor de correspondencia.

\section{BIBLIOGRAFÍA}

1. Silverman AL, Hammel N, Khachatryan N, Sharpsten L, Medeiros FA, Liebmann JM, et al. Diagnostic accuracy of the Spectralis and Cirrus Reference Databases in differentiating between healthy and early glaucoma eyes. Ophthalmology. 2016;123(2):408-14.

2. Kotowski J, Wollstein G, Ishikawa H, Schuman JS. Imaging of the optic nerve and retinal nerve fiber layer: an essential part of glaucoma diagnosis and monitoring. Surv Ophthalmol. 2014;59(4):458-67.
3. Tham YC, Li X, Wong TY, Quigley HA, Aung T, Cheng CY. Global prevalence of glaucoma and projections of glaucoma burden through 2040: a systematic review and meta-analysis. Ophthalmology. 2014;121(11):2081-90.

4. Medeiros FA, Vizzeri G, Zangwill LM, Alencar LM, Sample PA, Weinreb RN. Comparison of retinal nerve fiber layer and optic disc imaging for diagnosing glaucoma in patients suspected of having the disease. Ophthalmology. 2008;115(8):1340-6.

5. Chen TC. Spectral domain optical coherence tomography in glaucoma: qualitative and quantitative analysis of the optic nerve head and retinal nerve fiber layer (an AOS thesis). Trans Am Ophthalmol Soc. 2009;107:254-81.

6. Budenz DL, Chang RT, Huang X, Knighton RW, Tielsch JM. Reproducibility of retinal nerve fiber thickness measurements using the stratus TCO in normal and glaucomatous eyes. Invest Ophthalmol Vis Sci. 2005;46(7):2440-3.

7. Yalvac IS, Altunsoy M, Cansever S, Satana B, Eksioglu U, Duman S. The correlation between visual field defects and focal nerve fiber layer thickness measured with optical coherence tomography in the evaluation of glaucoma. J Glaucoma. 2009;18(1):53-61.

8. Pinilla I, Garcia-Martin E, Idoipe M, Sancho E, Fuertes I. Comparison of retinal nerve fiber layer thickness measurements in healthy subjects using Fourier and Time domain optical coherence tomography. J Ophthalmol. 2012;2012:107053.

9. Rolle T, Briamonte C, Curto D, Grignolo FM. Ganglion cell complex and retinal nerve fiber layer measured by Fourier-domain optical coherence tomography for early detection of structural damage in patients with preperimetric glaucoma. Clin Ophthalmol. 2011;5:961-9.

10. Chang RT, Knight OJ, Feuer WJ, Budenz DL. Sensitivity and specificity of time-domain versus spectral-domain optical coherence tomography in diagnosing early to moderate glaucoma. Ophthalmology. 2009;116(12):2294-9.

11. Crowston JG, Hopley CR, Healey PR, Lee A, Mitchell P; Blue Mountains Eye Study. The effect of optic disc diameter on vertical cup-to-disc ratio percentiles in a population-based cohort: The Blue Mountains Eye Study. Br J Ophthalmol. 2004;88(6):766-70.

12. Poon LY, Solá-del Valle D, Turalba AV, Falkenstein IA, Horsley M, Kim JH, et al. The ISNT rule: How often does it apply to disc photographs and retinal nerve fiber layer measurements in the normal population? Am J Ophthalmol. 2017:184:19-27.

13. Lin SC, Singh K, Jampel HD. Optic nerve head and retinal nerve fiber layer analysis: a report by the American Academy of Ophthalmology. Ophthalmology. 2007;114:1937-49. 